2020-04-21

\title{
Patient With Questions About Cancer Risk
}

Deborah O. Himes

Brigham Young University - Provo, deborah-himes@byu.edu

Jennie Vagher

Follow this and additional works at: https://scholarsarchive.byu.edu/facpub

Part of the Family Practice Nursing Commons, and the Other Nursing Commons

\section{Original Publication Citation}

Himes, D. O., \& Vagher, J. (2020). Patient With Questions About Cancer Risk. The Journal for Nurse Practitioners, 16(7), e109-e112.

\section{BYU ScholarsArchive Citation}

Himes, Deborah O. and Vagher, Jennie, "Patient With Questions About Cancer Risk" (2020). Faculty Publications. 5099.

https://scholarsarchive.byu.edu/facpub/5099 
Case Challenge

\title{
Patient With Questions About Cancer Risk
}

\author{
Deborah O. Himes, PhD, ANP-BC, Jennie Vagher, MS, CGC
}

\begin{abstract}
A B S T R A C T
Primary care nurse practitioners routinely care for patients with personal or family histories of cancer. Approximately $5 \%$ to $10 \%$ of all cancers are related to hereditary cancer syndromes (HCSs), ${ }^{1}$ which cause an increased risk for developing more cancers and cancers at earlier ages than the general population. Nurse practitioners in primary care must become comfortable with identifying patients at risk for HCSs. Ordering genetic tests can be a challenge because the number of genetic tests available is growing at a rapid pace. This case highlights a woman who survived breast cancer at age 25 and basal cell carcinoma at age 33 .
\end{abstract}

๑) 2020 Elsevier Inc. All rights reserved.

\section{Case Introduction}

A 35-year-old woman presents to her primary care nurse practitioner (NP) for a routine physical exam.

\section{Chief Complaint}

The patient expresses concern at having had 2 cancers at young ages. She reports having "negative BRCA test" but wonders if there is more testing she should consider.

\section{Past Medical and Surgical History}

The patient was diagnosed with breast cancer, at age 25 . She received bilateral mastectomies with reconstruction and adjuvant chemotherapy. She took chemoprevention medications for 2 years but discontinued because of side effects. She underwent BRCA1 and $B R C A 2$ genetic testing at age 26 in 2010; results were indeterminate negative (no mutation found). She had a basal cell carcinoma removed at age 33 in 2017.

Additional history includes endometriosis with endometrial ablation, ovarian cysts with total abdominal hysterectomy and bilateral salpingo-oophorectomy (TAH-BSO) at age 28, depression, and anxiety.

\section{Social History}

She is married with no children. She never used tobacco. She lived near a beach during childhood, worked as a lifeguard, and participated on swim team during high school. She reports spending a lot of time outdoors without sunscreen. She currently works in sales with no hazardous material exposure.

\section{Family History}

Her mother, age 73, survived breast cancer at 59. Her mother's history includes a large thigh lipoma surgically removed, uterine fibroids, thyroid nodules, and macrocephaly. Her maternal grandmother died at 80 , surviving colon cancer at 53 and breast cancer at 61. Her maternal uncle (71) survived 3 melanomas; maternal aunt (75) and other maternal uncles $(66,63)$ are all living with no cancer history. Her older brother, age 43 , survived a benign, solid brain tumor removed at 28 and has macrocephaly. Two older sisters (ages 51 and 43 ) have no pertinent history.

\section{Review of Systems}

General: She denies fever, chills, fatigue, weakness, or weight loss.

Lymph: She denies swollen nodes.

Skin: She denies rash or new lesions but states that she bruises easily.

HEENT: She denies headache, hearing or vision problems, oral lesions, or sore throat.

Pulmonary: She denies shortness of breath, orthopnea, cough, light-headedness, or dizziness.

Cardiac: She denies chest pain, or palpitations.

Abdomen: She denies abdominal pain, vomiting, reports normal stool and bowel patterns but complains of occasional nausea. Genitourinary: She denies dysuria or vaginal problems. She is in surgical menopause without estrogen replacement due to history of breast cancer.

\section{Physical Exam}

Vital signs: Her vitals are within normal limits. 
Neuro: She is alert and oriented.

Skin: She has no concerning lesions or rashes. The scar from basal cell removal is well healed.

Neck: Her neck is supple with no thyromegaly or lymphadenopathy.

Pulmonary: Her respirations are even, unlabored, and clear to auscultation.

Cardiac: S1 and S2 are noted with no murmurs, rubs, or gallops.

Abdomen: Her abdomen is round, soft, and nontender.

Musculoskeletal: She has a steady gait, with normal range of motion and strength in all extremities.

Genitourinary: Her cervix, uterus, ovaries, and adnexa surgically absent. The vaginal mucosa is pale and dry.

\section{Diagnostic Studies}

All labs and colonoscopy/esophagogastroduodenoscopy are normal.

\section{Questions to Consider}

1. What hereditary cancer syndromes (HCSs) are in the differential diagnosis and why?

2. What testing could be ordered?

3. What pretest information should be shared with this patient?

4. What are appropriate referral sources for this patient?

If you believe you know the answers to the following questions, then test yourself and refer to page $\mathrm{e} 111$ for the answers. 


\section{Patient With Questions About Cancer Risk (continued from page e110)}

\section{Case Challenge Questions and Answers}

1. What HCSs are in the differential diagnosis and why?

a. Hereditary breast and ovarian cancer (HBOC) syndrome caused by pathogenic variants (PVs) in BRCA1/2 genes. Earlyonset breast cancer is a red flag for HBOC. Although this patient reported $B R C A 1 / 2$ testing was negative, further investigation is warranted. Genetic tests change over time because testing techniques, genetic testing access, and variant databases improve. Early lab testing for BRCA1/2 and current direct-to-consumer lab testing often missed/miss large genomic alterations, such as translocations and deletions or duplications. Genetic testing of BRCA1/2 in 2006 for this patient included sequencing only, large rearrangement testing was not performed. Testing for large genomic rearrangements is now standard of care, ${ }^{1}$ thus retesting BRCA1/2 and including testing for rearrangements could be helpful. Approximately, $1 \%$ to $2 \%$ of those with negative $B R C A 1 / 2$ sequencing testing will have a large genomic rearrangement. Approximately $6 \%$ to $10 \%$ of those with PVs in BRCA1/2 will be due to large rearrangements. ${ }^{1}$ Although this patient is at low risk for future breast cancer (having already undergone bilateral mastectomies reduced her risk by $90 \%$ ) and the risk for ovarian cancer following premenopausal oophorectomy is $1 \%$ to $2 \%$, other cancers are associated with BRCA1/2 PVs. This patient meets National Comprehensive Cancer Network (NCCN) testing criteria for HCSs guidelines because her breast cancer onset was $\leq 45$ years of age. ${ }^{1}$

b. PTEN-hamartoma tumor syndrome (PHTS), Cowden syndrome, caused by PVs in the PTEN gene. Women with PHTS have up to an $85 \%$ lifetime risk for developing breast cancer and age of onset is typically $<50$ years. This patient does not meet testing criteria for PHTS, having only 1 major criteria (breast cancer); however, her mother would meet criteria. ${ }^{1}$ PHTS has also been associated with multiple hamartomatous tumors as well as adult Lhermitte-Duclos disease (dysplastic gangliocytoma of the cerebellum). The patient's brother had a brain tumor of unknown type. Although the patient herself does not meet NCCN testing criteria, new symptoms may develop over time, and she could in the future. Testing the person who meets NCCN criteria is ideal; however, if the mother cannot be tested for PHTS, it would not be unreasonable to test this patient.

c. Li-Fraumeni syndrome (LFS) caused by PVs in the TP53 gene. LFS is a highly penetrant cancer syndrome with $50 \%$ of women developing some type of cancer by age 31 years and nearly $100 \%$ penetrance by age 60 years. ${ }^{2}$ Family history consistent with LFS includes early-onset breast cancer and a brother with a brain tumor. With many family members who have lived to old age without cancer, the family history is not strongly suggestive of this syndrome. However, LFS should remain in the differential because it has a $20 \%$ de novo rate, ${ }^{3}$ meaning in $20 \%$ of those with LFS developed the PV as a fertilized egg in early embryogenesis. Another mechanism of inheritance that does not exhibit family history suggestive of LFS is mosaicism. With mosaicism, the PV develops during embryogenesis, so only a portion of the cells contain the PV (between $20 \%$ and $50 \%$ variant allele frequency). ${ }^{4}$ Gonadal mosaicism occurs when a parent's germ cell developed the $\mathrm{PV}$, which was transmitted to the embryo causing LFS. ${ }^{2}$ The $\mathrm{NCCN}^{1}$ recommends consideration of TP53 testing in women with early-onset breast cancer (age $\leq 30$ years at diagnosis) regardless of other family history.

d. Hereditary breast cancer caused by PVs in the ATM, CHEK2, and PALB2 genes. PVs in the ATM, CHEK2, and PALB2 genes confer a moderately increased risk for breast cancer (typically a 2 - to 5 -fold increase in relative risk). ${ }^{5}$ Approximately $2 \%$ to $5 \%$ of those referred for panel testing will have a PV identified in a moderate risk breast cancer gene. ${ }^{5}$ In contrast to those with PVs in BRCA1/2, the ages of breast cancer seen in women with moderate risk gene PVs vary significantly, ranging from early onset to ages more similar to the general population. Family history suggestive of an underlying moderate risk gene PV includes the patient's mother's and the patient's histories of breast cancer. The patient would meet NCCN testing criteria because her age of breast cancer onset was $<45$.

2. What testing could be ordered?

Next-generation sequencing (NGS) has increased the availability of genetic testing consisting of multiple genes for a variety of indications and allowed for multigene panel testing (MPT) to be integrated into clinical standard of care. MPT has been shown to identify more women with PVs than single-gene testing. MPT is a more cost-effective approach to genetic testing, especially in the case of this patient, who meets criteria for multiple HCSs. If NPs choose to order genetic tests, it is critical to investigate laboratory-billing practice before ordering tests. Some laboratories will bill for each individual gene on the panel; this practice can lead to large bills (in excess of $\$ 10,000$ ) for noncovered services. Other labs might bill for the BRCA codes, which are typically covered based on US Preventative Services Task Force, ${ }^{6}$ $\mathrm{NCCN},{ }^{1}$ or insurance-specific guidelines and will not bill the patient separately for other tests covered in the panel. Many patients billed in this way will pay less than $\$ 100$ if they meet testing criteria.

3. What pretest information should be shared with this patient? The American Society of Clinical Oncology ${ }^{7}$ and National Society of Genetic Counselors (NSGC) ${ }^{8}$ have outlined important elements of pretest counseling for single-gene and multigene testing. Clinicians should inform patients about the implications of genetic testing before ordering the test. When panel tests are performed, positive findings may be unexpected or lack specific guidelines for management. ${ }^{9}$ Specifically, this patient needs to understand what genes will be tested, that there could be psychological implications of testing (both benefits and risks), and the implications of positive results as well as uninformative results or variants of uncertain significance. ${ }^{7}$ For patients who are unaffected with cancer, risks of genetic discrimination and implications of the Genetic Information Non-discrimination Act (GINA) should be discussed. Patients should be counseled about potential implications for family members and the importance of sharing information with family members who may be at risk. If this patient still had reproductive organs, then she would be counseled about potential reproductive 
implications. ${ }^{7}$ A plan should be in place for reviewing test results and providing follow-up care and counseling. ${ }^{7}$ Finally, the HCSs discussed in this article require complex management. NPs should be familiar with community resources for patients with HCSs that require increased management. For example, those with LFS are recommended to undergo rapid sequence whole body magnetic resonance imaging (MRI) among other designated body MRIs and procedures, which takes careful coordination with expertise in LFS. ${ }^{2}$

4. What are appropriate referral sources for this patient?

If NPs feel unprepared to provide pretest counseling, order genetic tests, and interpret genetic test results, they should refer to a genetic counselor or medical geneticist. NSGC maintains a directory that patients and clinicians can use to locate genetic counselors near them. The NSGC directory helps clinicians to differentiate between both telemedicine and in-person consults.

\section{Conclusion}

This patient was not offered further genetic testing at her annual exam. Her NP assured her that basal cell carcinoma is common and that although her breast cancer onset was early, her risk for subsequent cancer was low because she had bilateral mastectomies and TAH-BSO. The NP was primarily concerned with BRCA1/2 mutations and felt the patient had already undergone adequate genetic testing.

Ten months after this visit, the patient found a dark mole on the back of her leg. Following biopsy, malignant melanoma was surgically removed. At that point, a MPT was ordered by her oncologist. Genetic test results revealed a PV in the TP53 gene (non-mosaic), which confers a diagnosis of LFS. All first-degree relatives underwent cascade testing and were negative, indicating that this was mostly likely a de novo event arising in the patient. One week later, the patient was diagnosed with non-small cell lung cancer after lung lesions were identified incidentally on a chest CT. Two primary tumors were removed from separate lobes, and lymph nodes were negative. No chemotherapy was recommended.

Given the results of this genetic testing, including the negative familial testing, the patient experienced an increase in anxiety and depression. She did feel reassurance knowing that no one else in the family was affected with LFS and that no testing for children needed to be offered. However, she also experienced feelings of isolation and distress, which is well documented in those with LFS. $^{10}$ Careful follow-up for this patient was recommended, including referral to a specialized management clinic. Clinics such as this provide expertise about management for LFS, specifically relating to frequency and interval of screening, as well as provide psychosocial support by means of genetic counseling or clinical psychologists with access to other mental health providers (e.g., social workers or psychiatrists) as needed. This patient was referred to a psychiatrist for discussion of her current anxiety and depression medication after meeting with the LFS clinic's clinical psychologist.

With knowledge of a TP53 PV, this patient is now following the LFS screening protocol, which will include annual rapid sequence whole body MRI, brain MRI, mole mapping, upper and lower endoscopies, and intense follow-up of any abnormal symptoms. Additionally, she now has access to an LFS support group and is working on coping with her risk for future cancer.

The NP in this case missed an opportunity to order genetic testing or make a genetics referral to identify a HCS 1 year before further cancers were identified. It is fortunate in this case that the patient was performing self-skin exams and identified early mole changes. Early diagnosis was key for this patient as a later diagnosis of melanoma could have been devastating. It was also fortunate that her lung cancer was identified as an incidental finding during a computed tomography and was treated early before symptoms arose.

The NP may have been falsely reassured because her patient was receiving regular follow-up with her oncologist. The NP may have assumed that if further testing was to be completed, the oncologist would have ordered it. However, unbeknownst to the NP, this patient had previously declined further testing, expressing concern about cost. At this annual exam, she was ready to consider updated genetic testing specifically because of her recent basal cell carcinoma diagnosis. NPs need to be aware of risk assessment, genetic counseling referral, genetic testing for HCSs, and follow-up management for those with HCSs.

\section{References}

1. National Comprehensive Cancer Network. Genetic/Familial High-Risk Assessment: Breast, Ovarian, and Pancreatic. NCCN Clinical Practice Guidelines in Oncology (NCCN Guidelines) 2020; Version 1.2020. December 4, 2019. https:// www.nccn.org/professionals/physician_gls/pdf/genetics_bop.pdf. Accessed January 14, 2020.

2. Kratz CP, Achatz MI, Brugières L, et al. Cancer screening recommendations for individuals with Li-Fraumeni syndrome. Clin Cancer Res. 2017;23(11):e38-e45.

3. PDQ Cancer Genetics Editoral Board. Genetics of Breast and Gynecologic Cancers $(P D Q \circledR)$ : Health Professional Version. PDQ Cancer Information Summaries. October 18, 2019. https://www.ncbi.nlm.nih.gov/books/NBK65767. Accessed January 14, 2020.

4. Weitzel JN, et al. Somatic TP53 variants frequently confound germ-line testing results. Genet Med. 2018;20(8):809-816.

5. Tung N, et al. Counselling framework for moderate-penetrance cancer-susceptibility mutations. Nat Rev Clin Oncol. 2016;13(9):581-588.

6. US Preventive Services Task Force, et al. Risk assessment, genetic counseling, and genetic testing for BRCA-related cancer: US Preventive Services Task Force recommendation statement. JAMA. 2019;322(7):652-665.

7. Robson ME, et al. American Society of Clinical Oncology policy statement update: genetic and genomic testing for cancer susceptibility. J Clin Oncol. 2015;33(31):3660-3667.

8. Berliner JL, et al. NSGC practice guideline: risk assessment and genetic counseling for hereditary breast and ovarian cancer. J Genet Couns. 2013;22(2): 155-163.

9. Lowstuter $\mathrm{K}$, et al. Unexpected $\mathrm{CDH} 1$ mutations identified on multigene panels pose clinical management challenges. JCO Precision Oncol. 2017;(1):1-12.

10. Peters JA, et al. Easing the burden: describing the role of social, emotional and spiritual support in research families with Li-Fraumeni syndrome. J Genet Couns. 2016;25(3):529-542.

Deborah O. Himes, PhD, ANP-BC, is an assistant professor at Brigham Young University, College of Nursing, Provo, UT. She can be contacted at Deborah-Himes@byu.edu. Jennie Vagher, MS, CGC, is a certified genetic counselor at Huntsman Cancer Institute, Salt Lake City, UT.

This work was supported by the Huntsman Cancer Foundation and the National Cancer Institute of the National Institutes of Health under award no. P30CA042014, which supports the Genetic Counseling Shared Resources.

In compliance with national ethical guidelines, the authors report no relationships with business or industry that would pose a conflict of interest. 